
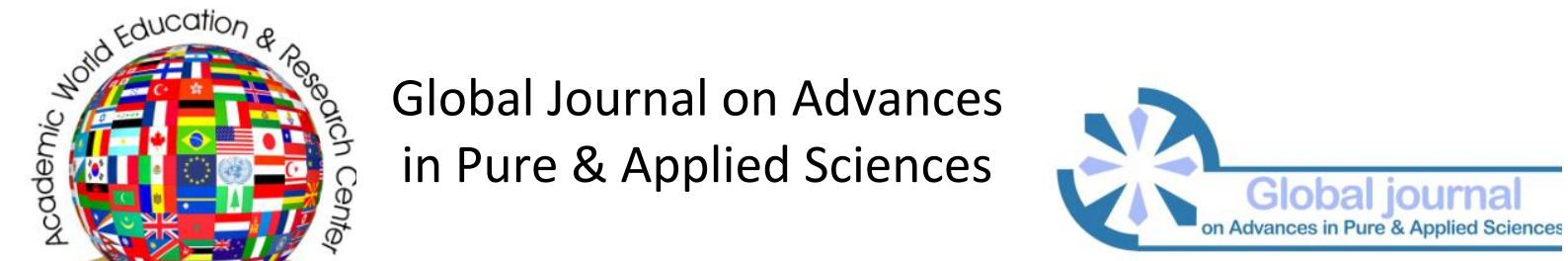

Issue 7 (2016) 01-12

Selected Paper of 2nd World Conference on Health Sciences (H-SCl 2015)

30 April-02 May 2015 Efes Sürmeli Hotel \& Convention Center - İzmir, Kuşadası, Turkey

\title{
Morphological quantification of myocardial pathology in the Zucker diabetic fatty rat
}

Abdulwahab Abuderman*, Physiology College of Medicine, Salman bin Abdulaziz University, Kharj, Saudi Arabia.

\section{Suggested Citation:}

Abuderman, A. (2016). Morphological quantification of myocardial pathology in the Zucker diabetic fatty rat, Global Journal on Advances in Pure \& Applied Sciences. [Online]. 07, pp 01-12. Available from: www.propaas.eu

Received October 16, 2014; revised December 27, 2014 ; accepted February 06, 2015.

Selection and peer review under responsibility of Prof. Dr. Fahrettin Sadikoglu, Near East University.

(C2016 Academic World Education \& Research Center. All rights reserved.

\begin{abstract}
Zucker Diabetic Fatty rat is an animal model that demonstrates disease progression in terms of complications which are similar to those seen in patients with Type 2 diabetes. The objective of the current study was to employ light and electron microscopy to quantify changes to the myocardial microvasculature and cardiomyocytes in the myocardial tissue of ZDF rats and establish a mechanistic basis for alterations in cardiac function. Materials and Methods:ZDF rats and lean Zucker rats (control) were housed in groups according to their breed. The ZDF rats were supplied with diabetogenic chow (Purina 5008) while the lean Zucker rats had access to standard chow as recommended by the supplier. At 12-14 weeks of age, animals were weighed and sacrificed by cervical dislocation. A blood sample was obtained for determination of blood glucose, and lipid profile.Both samples from LAA and the apex of left ventricle were carefully dissected, divided into small sections then fixed, impeded, sectioned, stained and random sections were photographed and the images were assessed and quantified using Image Analyser Pro-Plus software, version 4.1. Arterioles, venules, intermediate sized vessels, and capillaries were directly counted within the highlighted area of myocardium under light microscope. Ultra-thin sections were imaged in a Tecnai 12 Biotwin transmission electron microscope at a magnification of x4200 and photographed by a camera with a black and white film to quantify different structures of myocardium. Results: Significant reductions in the total vessel, intermediate vessel and capillary density of LAA in the ZDF rats compared to controls were noticed $(P=0.03)$. As well as a significant increase in the transverse diameter of cardiomyocytes in the ZDF rats compared to controls of LV $(P=0.049)$. There were significant
\end{abstract}

* ADDRESS FOR CORRESPONDENCE: Abdulwahab Abuderman, Physiology College of Medicine, Salman bin Abdulaziz University, Kharj -Saudi Arabia. E-mail address: a.abuderman@sau.edu.sa / Tel.: 00966-115886310 
increase in the basement membrane area of distal myocardial capillaries of both left atrium appendage and left ventricle in the ZDF rats compared to controls $(P=0.008)$.

Conclusion: Early evidence of cardiomyocyte hypertrophy with a reduction in atrial vascular density and evidence of early structural changes in myocardial capillaries in the ZDF rats was noticed. These changes indicate the presence of microangiopathy in the heart of ZDF rats which is surprisingly more prominent in the LAA.

Keywords: Zucker Diabetic Fatty (ZDF), Type 2 diabetes mellitus, Left Atrial Appendage, Left ventricle, Cardiomyocyte, Myocardial capillaries.

\section{Introduction}

A number of animal models of Type 2 diabetes mellitus (DM) such as monkeys [1], mice [2] and rats [3] have been used to study diabetes. A widely used model for obesity and Type 2 diabetes is the Zucker Diabetic Fatty (ZDF) rat. The ZDF rat is an inbred rat model and was established in 1987 after several inbreeding cycles of selected pairs of Zucker rats with diabetic rats [4]. All homozygous recessive animals $(f a / f a)$ supplied with standard rodent diet (Purina 5008) develop obesity, diabetes and its related complications, while homozygous dominant $(+/+)$ and heterozygous animals $(\mathrm{fa} /+)$ are lean and do not develop diabetes and can, therefore, be used as controls [4]. In these rats a gene mutation occurs in the extracellular domain of the leptin receptor protein [5] and results in shorteningof this receptor (Suhetal), leading to a defect in its interaction with leptin [6]. This impairs leptin's appetite suppressing effects [7] and the ZDF rats therefore develop hyperphagia, obesity, hyperinsulinaemia, insulin resistance, hyperglycaemia, hypertriglyceridaemia and hypercholesterolaemia [8,9]. In the first 6 months, obese Zucker rats gain 500 grams more weight compared with lean Zucker rats, after this time weight gain is equivalent [10]. The development of diabetes in ZDF rats closely resembles the pathogenesis of Type 2 diabetes in humans [9]. Obesity appears at an early stage with insulin resistance and hyperinsulinaemia, but with normal blood glucose [11, 13]. At 7-8 weeks, insulin levels fall and are unable to overcome hepatic glucose production leading to the development of hyperglycaemia. Insulin levels continue to fall and by around 22 to 24 weeks of age, they were below level of age-matched Zucker lean rats, due to pancreatic $b$ cells exhaustion $[8,11,12]$. The full picture of diabetes is complete around 12 weeks of age [6]. The majority of studies on the heart of the ZDF rat have focused on functional abnormalities. To date there are very few studies which have assessed structural changes [14, 17].

To our knowledge, no studies have examined vascular alterations in the myocardium of the obese ZDF rat; rather, they have focused on skeletal muscle, nerve, or kidney [18, 19].

It is important to note that although the ZDF rats develop hyperinsulinaemia and hyperglycaemia, the severity of the abnormalities varies between animals and diabetic patients [17]. Thus features of diabetic cardiomyopathy between patients and ZDF rats will undoubtedly differ but have not been evaluated [20].

The objective of the current study was to employ light and electron microscopy to quantify changes to the myocardial microvasculature and cardiomyocytes in the myocardial tissue of ZDF rats and establish a mechanistic basis for alterations in cardiac function.

\section{Materials and methods}

\subsection{Animals}

Five male ZDF $f a / f a$ rats and five male lean Zucker rats (control) were obtained from Charles River Laboratories at 6 weeks of age. They were housed in groups according to their breed and kept in an artificial lighting regime at room temperature. Both group of animals were given tap water and fed ad libitum. The ZDF rats were supplied with diabetogenic chow (Purina 5008) while the lean Zucker rats 
had access to standard chow as recommended by the supplier. All procedures were in accordance with the United Kingdom Animal (Scientific Procedures) act 1986. At 12-14 weeks of age, animals were weighed and sacrificed by cervical dislocation. A blood sample was obtained immediately after sacrifice for determination of serum levels of blood glucose, cholesterol, triglycerides, and HDL.

\subsection{Tissue processing}

Both samples from LAA and the apex of left ventricle were carefully dissected, divided into small sections and fixed in $2.5 \%$ glutaraldehyde in $1 \%$ cacodylate buffer ( $\mathrm{pH} 7.4)$. They were then secondarily fixed in $1 \%$ Osmium tetroxide. After that embedded in epon blocks and sectioned using Reichert mechanical advance ultra-microtome. $0.7 \mu \mathrm{m}$ sections were obtained for light microscopy and 0.08$0.09 \mu \mathrm{m}$ sections for electron microscopy. Semi-thin sections were stained with $1 \%$ Toluidine blue while ultra-thin sections were stained with MethanolicUranyl acetate and Lead citrate, the metallic and slightly radioactive dyes.

\subsubsection{Image processing and morphological procedures (light microscope)}

From each biopsy one suitable section was selected and photographed using a digital Kodak camera attached to a Leica WILD MPS32 photo light microscope (Leica, Cambridge, UK). 6 random areas from each section were visualized and photographed using Kodak image software.

Sections were photographed at 2 magnifications, (x20 and x40 magnifications). The $x 20$ lens was used to photograph most of the parameters while the $x 40$ lens was used to photograph the arterioles and venules. Images were assessed, and quantified using Image Analyser Pro-Plus software, version 4.1 (Media Cybernetics, Buckinghamshire UK), with a standard grid bar to calibrate the images. The following parameters were quantified:

- $\quad$ Arteriole, venule, intermediate vessel, and capillary density (no. $\left./ \mathrm{mm}^{2}\right)$ (x20)

- Arteriolar and venular wall to lumen area ratio (WLR) (x20)

- $\quad$ Transverse cardiomyocyte diameter $(\mu \mathrm{m})(\times 20)$

\subsubsection{Image processing and morphological procedures (Electron Microscopy)}

Ultra-thin sections were imaged in a Tecnai 12 Biotwin transmission electron microscope (FEl, Eindhoven, The Netherlands) set at $100 \mathrm{kV}$ accelerating voltage and a filament emission of 30 . The condenser settings and emissions were adjusted to achieve the highest contrast and 6 to 8 capillaries (microvessels without a complete layer of pericyte or smooth muscle surrounding the endothelial cells) favouring neither the large nor the small ones were photographed at a magnification of (x4200) using a camera with a black and white film. The film was processed in a dark room and an Apple Mac scanner and I-Mac computer were used to scan the negatives. The following parameters were quantified:

- Lumen area $\left(\mu \mathrm{m}^{2}\right)(\mathrm{X} 4200)$

- Endothelial outer membrane area $\left(\mu \mathrm{m}^{2}\right)(\mathrm{X} 4200)$

- Endothelial area $\left(\mu \mathrm{m}^{2}\right)(\mathrm{X} 4200)$

- Pericyte area $\left(\mu \mathrm{m}^{2}\right)(\mathrm{X} 4200)$ 
Abuderman, A. (2016). Morphological quantification of myocardial pathology in the Zucker diabetic fatty rat, Global Journal on Advances in Pure \& Applied Sciences. [Online]. 07, pp 01-12. Available from: www.propaas.eu

- Vessel area $\left(\mu \mathrm{m}^{2}\right)(\mathrm{X} 4200)$

- Basement membrane area $\left(\mu \mathrm{m}^{2}\right)$ (X4200)

- Endothelial cell profile (number) (X4200)

- Number of endothelial nuclei (number) (X4200)

- Number of pericyte nuclei (number) (X4200)

\subsection{Statistical Analysis}

Statistical analysis was performed using SPSS for Windows 14.0. All data are expressed as the Mean and standard error of mean (SEM). Data were compared between two groups by non-parametric Mann-Whitney U-test. Statistical significance was accepted for $\mathrm{P}<0.05$ (two-tailed).

\section{Results}

\subsection{Weight and metabolic values}

The weight was significantly increased in ZDF rats compared to lean rats ( $P=0.047)$. Blood glucose was also significantly increased in the ZDF rats compared to lean rats $(P=0.02)$. The triglyceride level $(P=0.002)$ and total cholesterol levels were significantly increased in ZDF rats compared to lean rats $(P=0.002)$. The HDL levels were significantly increased in the ZDF rats compared to lean rats $(P=0.001)$ (Table 1).

Table 1.Weight and blood values of ZDF and lean rats

\begin{tabular}{lccc|}
\hline & Controls & ZDF & $P$ Value \\
Weight $(\mathrm{g})$ & $(\mathrm{n}=5)$ & $(\mathrm{n}=5)$ & \\
& & & \\
& $323 \pm 7.9$ & $347.5 \pm 3.2$ & 0.047 \\
Blood glucose & $4.37 \pm 0.4$ & $19.1 \pm 3.3$ & 0.021 \\
$\begin{array}{l}\text { (mmol/l) } \\
\text { Triglycerides }\end{array}$ & & \\
$\begin{array}{l}\text { (mmol/l) } \\
\text { Cholesterol }\end{array}$ & $1.77 \pm 0.1$ & $6.70 \pm 0.6$ & 0.002 \\
(mmol/l) & & & \\
$\mathrm{HDL}$ & $2.60 \pm 0.2$ & $4.19 \pm 0.2$ & 0.002 \\
(mmol/l) & $1.80 \pm 0.04$ & $3.60 \pm 0.17$ & 0.001 \\
\hline
\end{tabular}




\subsection{LAA \& left ventricle morphometry: Light microscopy}

There were highly significant reductions in the total vessel, intermediate vessel and capillary density of LAA in the ZDF rats compared to controls $(P=0.03)$ (Table2). Although the arteriole and venule WLR of LAA were increased this was not significant between the two groups $(P=0.25,0.91)$. The tissue section showed hypertrophic cardiomyocytes in the LAA in diabetic Zuckerrat compared to control (Figure 1). There was a significant increase in the transverse diameter of cardiomyocytes in the ZDFrats compared to controls of $\operatorname{LV}(P=0.049) \quad$ (Figure 2). It was appeared that, there were no difference between the two groups for arteriole, venule, intermediate vessel, capillary and total vessel densities of LV respectively $(P=0.13,0.33,0.93,0.7,0.79)$

Table 2. Vessel density of LAA in the control and the ZDF rats

\begin{tabular}{llllll}
\hline Parameter & \multicolumn{3}{l}{ Controls } & \multicolumn{3}{l}{ ZDF } & $P$ value \\
\hline Arteriole density & 7.6 & \pm 1.5 & $4.1 \pm 1.4$ & 0.22 \\
Venule density & 38.3 & \pm 10.6 & 34.1 & \pm 2.8 & 0.84 \\
$\begin{array}{l}\text { Intermediate } \\
\text { vessel density }\end{array}$ & 90.8 & \pm 13.5 & 47.3 & \pm 8.7 & 0.03 \\
$\begin{array}{l}\text { Capillary density } \\
\begin{array}{l}\text { Total vessel } \\
\text { density }\end{array}\end{array}$ & $467.7 \pm 93.3$ & & & \\
\hline
\end{tabular}
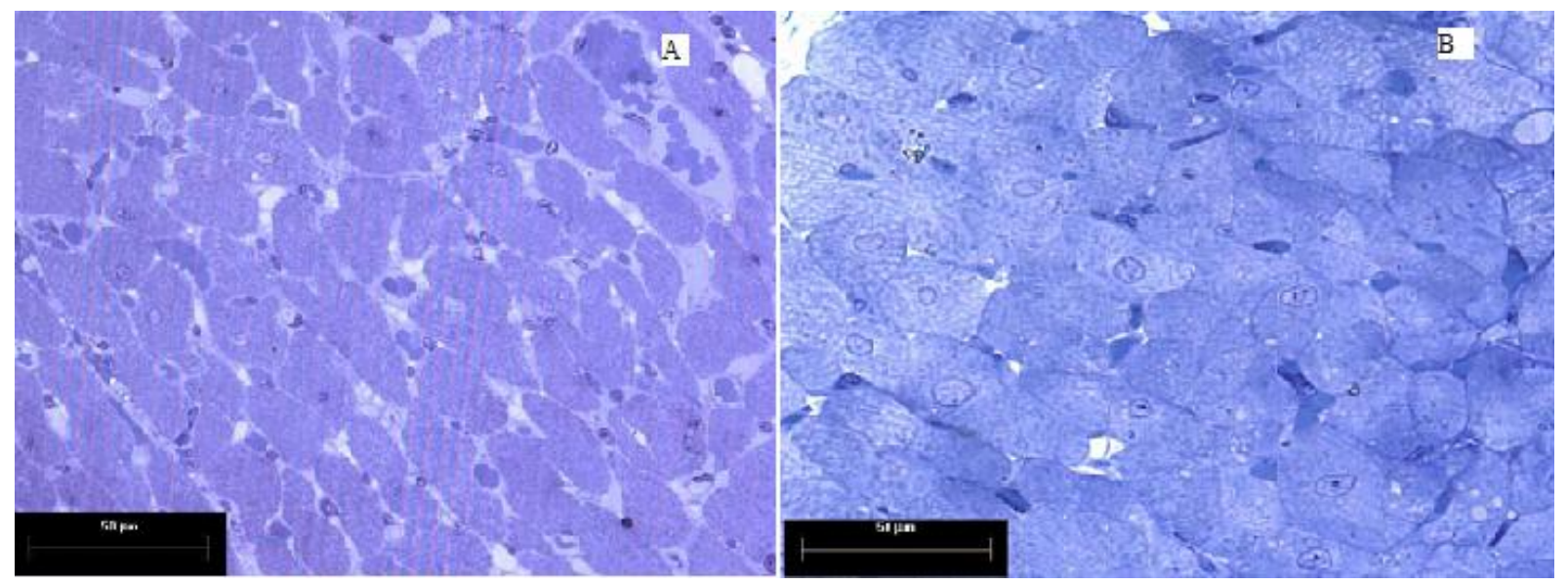

Figure 1. Light microscopic section of LAA from A- a lean Zucker rat. B- a ZDF rat 


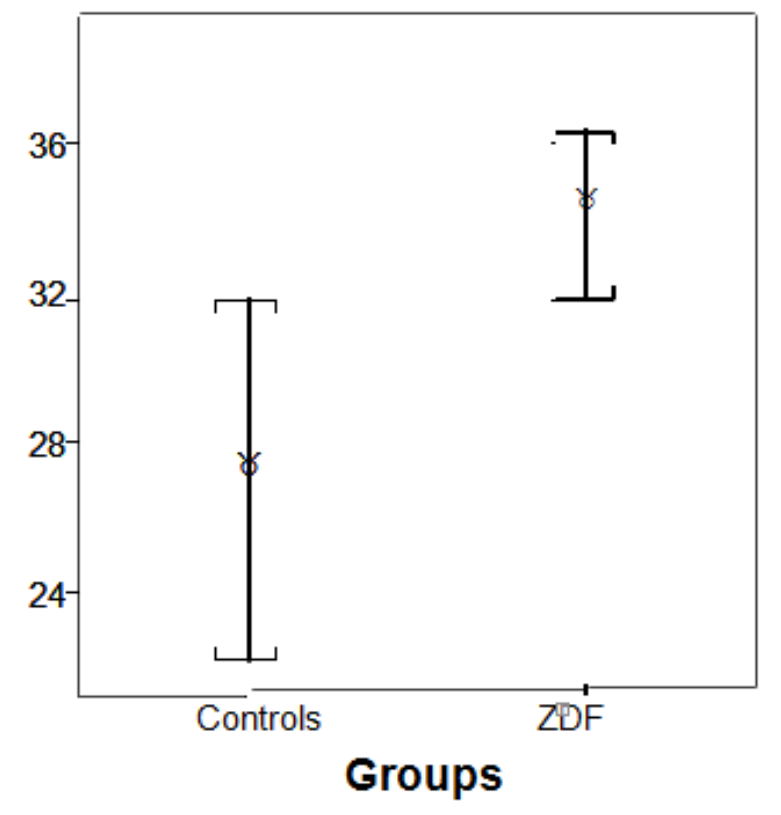

Figure 2.Transverse diameter of cardiomyocytes of LV in control and the ZDF rats.

\subsection{Pathology of distal myocardial capillaries in LAA and LV: Electron microscopy}

LAA: The lumen area was significantly reduced in ZDF rats compared to controls $(P=0.008)$. There was a significant increase in the basement membrane area of distal myocardial capillaries in the ZDF rats compared to controls $(P=0.008)$. Vessel area of the distal myocardial capillary did not show a significant difference between the two groups $(P=0.69)$. There was no significant difference found in capillary endothelial area, endothelial cell profile, Number of endothelial nuclei, capillary pericyte area and pericyte nuclei between the two groups $(P=0.55, P=0.42, P=0.22, P=0.10$ and $P=0.84)$ respectively. (Figure 3)

LV: There was no significant difference in lumen area, vessel area, capillary endothelial area, endothelial cell profile, Number of endothelial nuclei, capillary pericyte area and pericyte nuclei of the distal myocardial capillary between the two groups $(P=0.55, P=0.84, P=0.69, P=0.69, P=1.00, P=0.84$ and $P=0.55$ ) respectively. There was a significant increase in the basement membrane area of the distal myocardial capillary in ZDF rats compared to controls $(P=0.008)$. (Figure 4$)$ 

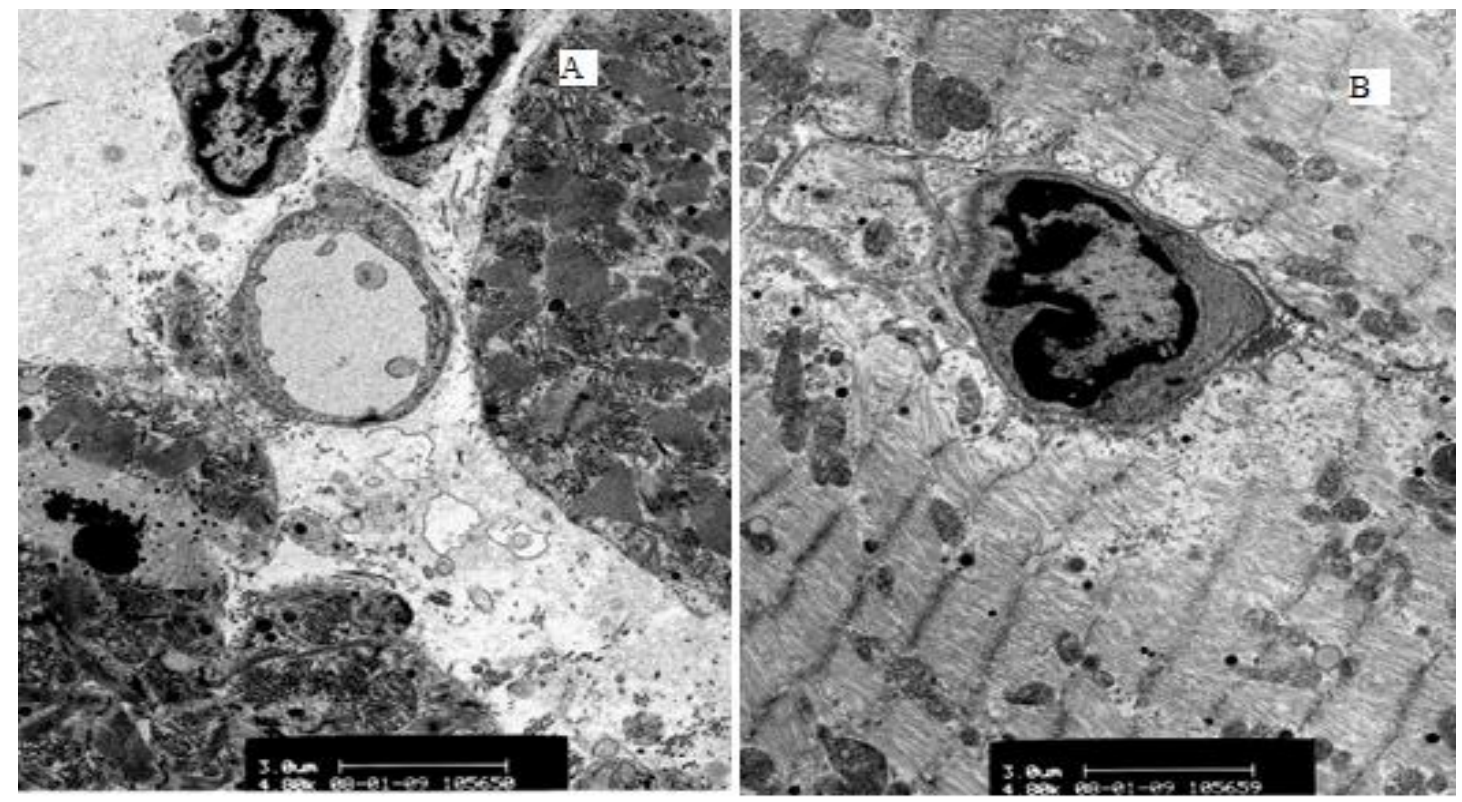

Figure 3. Electronmicrograph showing a myocardial capillary from the LAA of : A- a lean Zucker rat. B- ZDF rats.
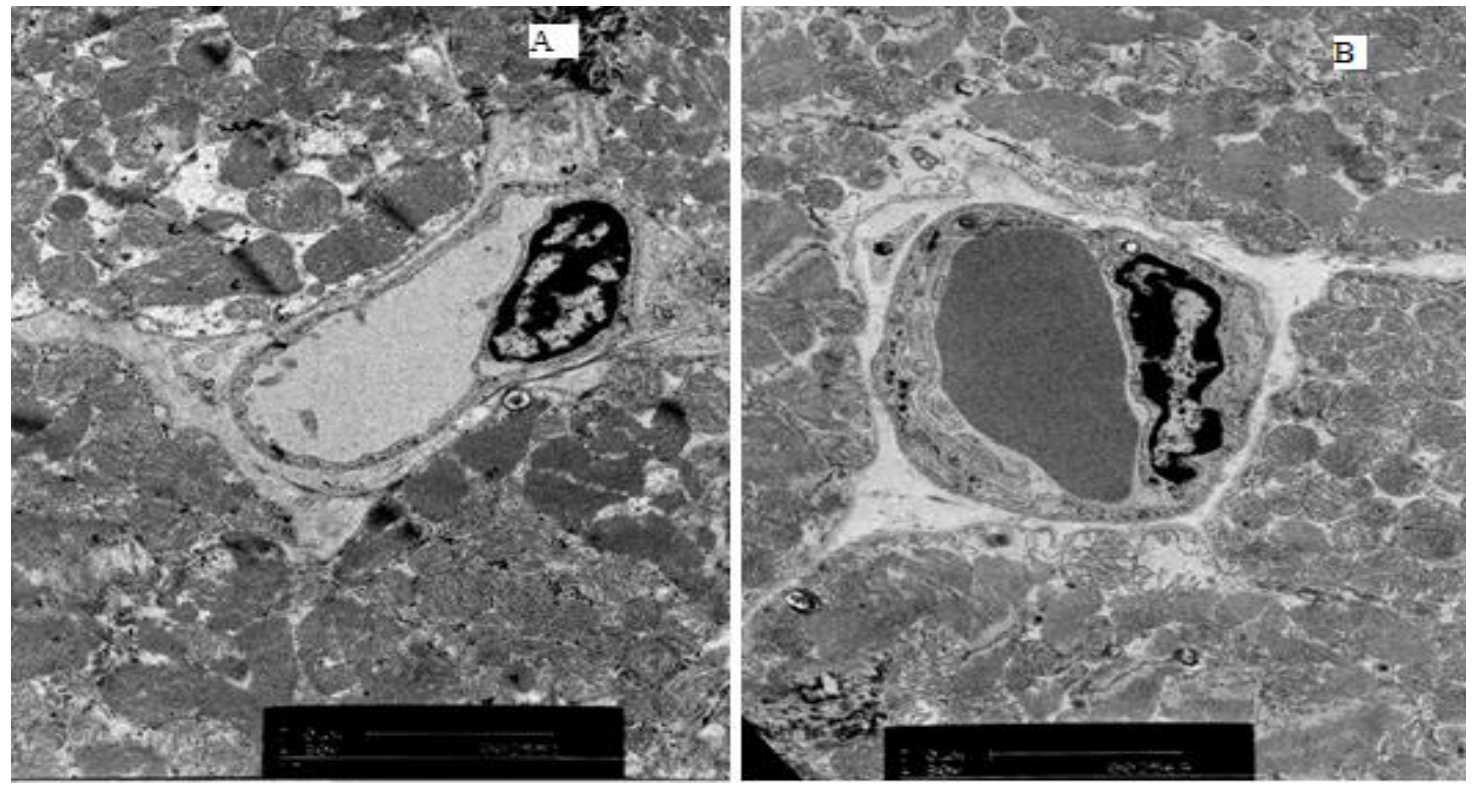

Figure 4. Electronmicrograph showing a myocardial capillary from the LV of: A- a lean Zucker rat. B- ZDF rats.

\section{Discussion}

Animal models of diabetes have been extensively used in diabetes research. Today experimental animals are used but with considerable ethical and legal restrictions [21]. The ZDF rat is an animal model that demonstrates disease progression in terms of complications which are similar to those seen in patients with Type 2 diabetes. The animals in our study were between 12 to 14 weeks of age, 
and they had developed all the metabolic abnormalities associated with Type 2 diabetes. Thus they demonstrate a significant increase in weight, blood glucose (5 fold increase), triglycerides (6 fold increase) and cholesterol ( 2 fold increase), but paradoxically they had an increased HDL (3 fold increase). The very high HDL level is commonly observed in this animal model and clearly differs from the situation expected in the obese diabetic patient, where HDL is low. This could arguably result in lesser atherosclerosis and macrovascular pathology in this model. Atherosclerosis is of course difficult to demonstrate in many animal models without additional feeding of high cholesterol diets. The ZDF rat may provide an opportunity to study the effects of some but not all the metabolic lipid abnormalities of Type 2 diabetes, a disease in which macrovascular and cardiac complications account for a major part of its morbidity and mortality. Thus even in the 20 week old ZDF rat an additional 4 weeks of cholesterol feeding is required to significantly exacerbate myocardial injury produced by coronary occlusion-reperfusion and to impair endothelial function in the coronary bed [22].

Few studies have quantified myocardial pathology, especially in the ZDF rat. In studies of ventricular specimens from patients with diabetes, pathological changes include a decrease in small vessel density, a reduction in the vessel luminal area, an increase in the vessel wall area and both hypertrophy and atrophy of cardiomyocytes with myocardial lipid deposition ${ }^{23}$ and autonomic nerve fibre loss $[24,25]$. The results of our study in the ZDF rats show some trends which are in keeping with the changes seen in humans. We showed a significant reduction in intermediate vessel, capillary and total vessel density in the LAA of diabetic animals compared to controls. In accord with our findings, Toblliet al [26] showed a significant reduction in the myocardial capillary density as well as VEGF expression of 8 month old hyperglycaemic obese Zucker rats (OZR) compared to lean littermates. In addition, there was a significant positive correlation between VEGF expression and the number of vessels in all groups [26]. The expression of mRNA and protein for VEGF (25\%) and its receptors VEGFR $_{2}(25 \%)$ but not VEGFR 1 have also been shown to be reduced in the heart of the 14 week ZDF rats [27]. Thus, the reduction in vascular density observed in ZDF rats could be explained by the reduction of VEFG expression.

Both hypertrophy and atrophy has been demonstrated in cardiomyocytes of diabetic hearts. In this study cardiomyocyte diameter was increased in the LAA and was most pronounced in the left ventricle of the ZDF rat. In a recent study of ZDF rats after 2 months of diabetes a twofold increase in cardiomyocyte volume was observed and quantified by defining the cardiomyocyte width (CMW) and then deriving volume by determining cardiomyocytelength $(\mathrm{CML})$ and calculating $\mathrm{CMW} / 2 \times \pi \times \mathrm{CML}$. At the ultrastructural level there was evidence of perivascular fibrosis determined by measurement of the thickness of the collagen I area surrounding the vessels. Quantitative immunohistochemistry of areas positive for fibronectin and collagen I showed no increase in interstitial collagen I or fibronectin [15]. Cardiomyocyte hypertrophy has been demonstrated in newborn streptozocin-induced diabetic female rats which then disappears after 4 weeks [28]. A consequence of these structural alterations could be impaired cardiomyocyte relaxation which has been demonstrated in 22 but not 6 week old ZDF rats and which has been related to increased levels of O-linked attachment of N-acetylglucosamine (O-GIcNAc) and nucleocytoplasmic proteins [29]. In the 4-10 week streptozotocin (STZ)induced diabetic rat there is reduced viability of ventricular cardiomyocytes and the amplitude of contraction is significantly reduced [30].

In the current study we showed a significant increase in the basement membrane area of distal myocardial capillary in the ZDF rats compared to lean littermates in both the atrium and ventricle. In addition, we find a significant reduction in the capillary luminal area of the LAA in the ZDF rats compared to lean littermates. We were not aware of any other studies in the literature, which have quantified capillary structure in the myocardium of the ZDF rat. In other tissues of the ZDF rat previous studies have assessed retinal abnormalities and showed no gross abnormality of retinal capillaries on trypsin digests but there was evidence of endothelial cell hyperplasia and basement membrane thickening (mean thickness $21 \%$ greater in diabetic rats) [31]. In addition, recent studies have shown a significant increase in pericyte and endothelial cell apoptosis of retinal capillaries in ZDF rats, which was promoted by tumour necrosis factor-alpha [32]. In the kidney, studies have shown a significant 
thickening of the glomerular basement membrane in ZDF rats compared to lean littermates ${ }^{33}$. In addition, other studies have shown renal capillary density to be increased, both in the cortex and in the inner medulla at 8 weeks, but significantly decreased by 22 weeks in ZDF rats compared to lean littermates. This was associated with altered expression of vascular endothelial growth factor (VEGF), reduced expression of Flk-1, and neuropilin. With regard to interlobar arteries, acetylcholine-induced relaxation was not impaired at 8 weeks but was significantly attenuated at 22 weeks [34]. Similarly in the ZDF rat at 26 weeks, acetylcholine induced endothelium-dependent relaxation is impaired, yet blood pressure and perivascular sensory nerve CGRP content are normal [35]. However, recently the diabetic ZDF rat at 17-20 weeks of age has been shown to have altered endothelial function which was related to an alteration in the vascular myocyte large-conductance $\mathrm{Ca}^{2+}$-activated $\mathrm{K}^{+}$channels $(\mathrm{BK}(\mathrm{Ca}))$, but with no reduction in the beta(1) subunit [36]. Thickening of the capillary basement membrane has also been observed in the plantar muscle of 11 week old obese Zucker rats. However, at 11 to 18 weeks of age, the capillary basement membrane reduced in thickness in both lean and obese animals and the decline was greatest in OZRs. Thus, by 18 weeks of age, the capillary basement membrane of OZRs was only slightly thicker than that in lean littermates [19].

Many factors have been implicated in the development of capillary basement membrane thickening in diabetic microangiopathy in both humans and animal models. In diabetes, loss of myogenic response increases capillary pressure and blood flow and subsequently hydrostatic pressure which might lead to an increase in vessel wall permeability and activation of proliferative change particularly basement membrane thickening [37]. In kidney, studies have suggested that reduced expression and activity of collagenolytic enzymes, in association with hyperglycaemia-induced modulation of integrin expression, might play an important role in the development of glomerular basement membrane thickening [38]. Additionally, the regulation of matrix metalloproteinase (MMP) activity by tissue inhibitors of metalloproteinases (TIMP) might contribute to glomerular basement membrane thickening. Expression of TIMP-2, a specificinhibitor of MMP-2, was highly elevated in glomerular epithelial cells cultured in high glucose [39] and also in the kidneys of Type 1 diabetic rats [40]. Also oxidative stress/oxygen radicals might contribute to matrix accumulation and subsequent basement membrane thickening in diabetic nephropathy [41, 42]. A similar increase in the expression of matrix components has also been observed in the capillaries of diabetic retina $[43,44]$. Basement membrane of retinal capillaries of diabetic mice showed a significant increase in the $\alpha 1$ (IV) chain of collagen, as well as of the 81 and $y$ chains of laminin and of fibronectin, as early as 8 weeks following induction of diabetes, indicating increased matrix component expression [45].

The functional consequences of basement membrane thickening have been demonstrated in the skin vessel of Type 1 diabetic patients with nephropathy. They include a reduction in vascular distensiblility and an increase in minimal vascular resistance which result in limitation of adequate tissue perfusion [46].

In summary, we demonstrate early evidence of cardiomyocyte hypertrophy with a reduction in atrial vascular density in the ZDF rats. In addition, we show evidence of early structural changes in myocardial capillaries characterised by basement membrane thickening and a reduction in atrial capillary luminal area. These changes indicate the presence of microangiopathy in the heart of ZDF rats which is surprisingly more prominent in the LAA.

\section{Acknowledgments}

The author deeply thanksall who contributed in the success of this work.

\section{Conflict of Interest}

The authors certify that there was no conflict of interest. 
Abuderman, A. (2016). Morphological quantification of myocardial pathology in the Zucker diabetic fatty rat, Global Journal on Advances in Pure \& Applied Sciences. [Online]. 07, pp 01-12. Available from: www.propaas.eu

\section{References}

[1] Kim, S., Y., Johnson, M., A., McLeod, D., S., Alexander, T., Hansen, B., C., \& Lutty, G., A. (2005). Neutrophils are associated with capillary closure in spontaneously diabetic monkey retinas. Diabetes, 54, 15341542.

[2] Srinivasan, K., \& Ramarao, P. (2007). Animal model in type 2 diabetes research: An overview. Indian Journal of Medical Research, 125(3), 451.

[3] Kasiske, B. L., O'Donnell, M. P., \& Keane, W. F. (1987). The obese Zucker rat model of glomerular injury in type II diabetes. Journal of Diabetic Complications, 1(1), 26-29.

[4] Peterson, R. G., Shaw, W. N., Neel, M. A., Little, L. A., \& Eichberg, J. (1990). Zucker diabetic fatty rat as a model for non-insulin-dependent diabetes mellitus. ILAR Journal, 32(3), 16-19.

[5] Pickavance, L., Widdowson, P. S., King, P., Ishii, S., Tanaka, H., \& Williams, G. (1998). The development of overt diabetes in young Zucker Diabetic Fatty (ZDF) rats and the effects of chronic MCC-555 treatment. British journal of pharmacology, 125(4), 767-770.

[6] Suh, Y. H., Kim, Y., Bang, J. H., Choi, K. S., Lee, J. W., Kim, W. H., ... \& Jung, M. H. (2005). Analysis of gene expression profiles in insulin-sensitive tissues from pre-diabetic and diabetic Zucker diabetic fatty rats. Journal of molecular endocrinology, 34(2), 299-315.

[7] Terrettaz, J., \& Jeanrenaud, B. (1983). In Vivo Hepatic and Peripheral Insulin Resistance in Genetically Obese (fa/fa) Rats*. Endocrinology, 112(4), 1346-1351.

[8] Peterson, R. G., Shaw, W. N., Neel, M. A., Little, L. A., \& Eichberg, J. (1990). Zucker diabetic fatty rat as a model for non-insulin-dependent diabetes mellitus. ILAR Journal, 32(3), 16-19.

[9] Leonard, B. L., Watson, R. N., Loomes, K. M., Phillips, A. R. J., \& Cooper, G. J. (2005). Insulin resistance in the Zucker diabetic fatty rat: a metabolic characterisation of obese and lean phenotypes. Acta diabetologica, 42(4), 162-170.

[10] Schmidt, R. E., Dorsey, D. A., Beaudet, L. N., \& Peterson, R. G. (2003). Analysis of the Zucker Diabetic Fatty (ZDF) type 2 diabetic rat model suggests a neurotrophic role for insulin/IGF-I in diabetic autonomic neuropathy. The American journal of pathology, 163(1), 21-28.

[11] Corsetti, J. P., Sparks, J. D., Peterson, R. G., Smith, R. L., \& Sparks, C. E. (2000). Effect of dietary fat on the development of non-insulin dependent diabetes mellitus in obese Zucker diabetic fatty male and female rats. Atherosclerosis, 148(2), 231-241.

[12] Etgen, G. J., \& Oldham, B. A. (2000). Profiling of Zucker diabetic fatty rats in their progression to the overt diabetic state. Metabolism, 49(5), 684-688.

[13] Shibata, T., Takeuchi, S., Yokota, S., Kakimoto, K., Yonemori, F., \& Wakitani, K. (2000). Effects of peroxisome proliferator-activated receptor- $\alpha$ and- $\gamma$ agonist, JTT-501, on diabetic complications in Zucker diabetic fatty rats. British journal of pharmacology, 130(3), 495-504.

[14] Chatham, J. C., \& Seymour, A. M. L. (2002). Cardiac carbohydrate metabolism in Zucker diabetic fatty rats. Cardiovascular research, 55(1), 104-112.

[15] Fredersdorf, S., Thumann, C., Ulucan, C., Griese, D. P., Luchner, A., Riegger, G. A., ... \& Weil, J. (2004). Myocardial hypertrophy and enhanced left ventricular contractility in Zucker diabetic fatty rats. Cardiovascular Pathology, 13(1), 11-19.

[16] Wang, P., \& Chatham, J. C. (2004). Onset of diabetes in Zucker diabetic fatty (ZDF) rats leads to improved recovery of function after ischemia in the isolated perfused heart. American Journal of PhysiologyEndocrinology and Metabolism, 286(5), E725-E736.

[17] Poornima, I. G., Parikh, P., \& Shannon, R. P. (2006). Diabetic cardiomyopathy the search for a unifying hypothesis. Circulation research, 98(5), 596-605.

[18] Frisbee, J. C., Maier, K. G., \& Stepp, D. W. (2002). Oxidant stress-induced increase in myogenic activation of skeletal muscle resistance arteries in obese Zucker rats. American Journal of Physiology-Heart and Circulatory Physiology, 283(6), H2160-H2168.

[19] Lash, J. M., Sherman, W. M., \& Hamlin, R. L. (1989). Capillary basement membrane thickness and capillary density in sedentary and trained obese Zucker rats. Diabetes, 38(7), 854-860.

[20] An, D., \& Rodrigues, B. (2006). Role of changes in cardiac metabolism in development of diabetic 
Abuderman, A. (2016). Morphological quantification of myocardial pathology in the Zucker diabetic fatty rat, Global Journal on Advances in Pure \& Applied Sciences. [Online]. 07, pp 01-12. Available from: www.propaas.eu

cardiomyopathy. American Journal of Physiology-Heart and Circulatory Physiology, 291(4), H1489H1506.

[21] Rees, D. A., \& Alcolado, J. C. (2005). Animal models of diabetes mellitus.Diabetic medicine, 22(4), 359-370.

[22] Hoshida, S., Yamashita, N., Otsu, K., Kuzuya, T., \& Hori, M. (2000). Cholesterol feeding exacerbates myocardial injury in Zucker diabetic fatty rats.American Journal of Physiology-Heart and Circulatory Physiology, 278(1), H256-H262.

[23] Sharma, S., Adrogue, J. V., Golfman, L., Uray, I., Lemm, J., Youker, K., ... \& Taegtmeyer, H. (2004). Intramyocardial lipid accumulation in the failing human heart resembles the lipotoxic rat heart. The FASEB Journal, 18(14), 1692-1700.

[24] Adeghate, E. (2004). Molecular and cellular basis of the aetiology and management of diabetic cardiomyopathy: a short review. Molecular and cellular biochemistry, 261(1), 187-191.

[25] Fang, Z. Y., Prins, J. B., \& Marwick, T. H. (2004). Diabetic cardiomyopathy: evidence, mechanisms, and therapeutic implications. Endocrine reviews, 25(4), 543-567.

[26] Toblli, J. E., Cao, G., DeRosa, G., Di Gennaro, F., \& Forcada, P. (2004). Angiotensin-converting enzyme inhibition and angiogenesis in myocardium of obese Zucker rats. American journal of hypertension, 17(2), 172-180.

[27] Chou, E., Suzuma, I., Way, K. J., Opland, D., Clermont, A. C., Naruse, K., ... \& King, G. L. (2002). Decreased cardiac expression of vascular endothelial growth factor and its receptors in insulin-resistant and diabetic states a possible explanation for impaired collateral formation in cardiac tissue.Circulation, 105(3), 373-379.

[28] Takino, Y., Iwasaki, T., \& Suzuki, T. (1990). The cardiomyopathy in infants of streptozotocin-induced diabetic female rats. Japanese circulation journal, 54(12), 1554-1562.

[29] Fülöp, N., Mason, M. M., Dutta, K., Wang, P., Davidoff, A. J., Marchase, R. B., \& Chatham, J. C. (2007). Impact of Type 2 diabetes and aging on cardiomyocyte function and O-linked $\mathrm{N}$-acetylglucosamine levels in the heart. American Journal of Physiology-Cell Physiology, 292(4), C1370-C1378.

[30] Bracken, N., Howarth, F. C., \& Singh, J. (2006). Effects of Streptozotocin-Induced Diabetes on Contraction and Calcium Transport in Rat Ventricular Cardiomyocytes. Annals of the New York Academy of Sciences, 1084(1), 208-222.

[31] Danis, R. P., \& Yang, Y. (1993). Microvascular retinopathy in the Zucker diabetic fatty rat. Investigative Ophthalmology and Visual Science, 34, 2367-2371.

[32] Behl, Y., Krothapalli, P., Desta, T., DiPiazza, A., Roy, S., \& Graves, D. T. (2008). Diabetes-enhanced tumor necrosis factor- $\alpha$ production promotes apoptosis and the loss of retinal microvascular cells in type 1 and type 2 models of diabetic retinopathy. The American journal of pathology, 172(5), 1411-1418.

[33] Ward, B. J., \& Donnelly, J. L. (1994). The endothelial vesicular system in cardiac capillaries subjected to hypoxia. Microvascular research, 48(2), 143-150.

[34] Gealekman, O., Brodsky, S. V., Zhang, F., Chander, P. N., Friedli, C., Nasjletti, A., \& Goligorsky, M. S. (2004). Endothelial dysfunction as a modifier of angiogenic response in Zucker diabetic fat rat: amelioration with Ebselen.Kidney international, 66(6), 2337-2347.

[35] Pamarthi, M. F., Rudd, M. A., \& Bukoski, R. D. (2002). Normal perivascular sensory dilator nerve function in arteries of Zucker diabetic fatty rats*.American journal of hypertension, 15(4), 310-315.

[36] Burnham, M. P., Johnson, I. T., \& Weston, A. H. (2006). Reduced Ca2+-dependent activation of largeconductance $\mathrm{Ca2+-activated} \mathrm{K}+$ channels from arteries of Type 2 diabetic Zucker diabetic fatty rats. American Journal of Physiology-Heart and Circulatory Physiology, 290(4), H1520-H1527.

[37] Tooke, J. E. (1999). A pathophysiological framework for the pathogenesis of diabetic microangiopathy. Diabetic Angiopathy. Arnold: London, UK, 187-194.

[38] Tsilibary, E. C. (2003). Microvascular basement membranes in diabetes mellitus. The Journal of pathology, 200(4), 537-546.

[39] Kitsiou, P. V., Tzinia, A. K., Stetler-Stevenson, W. G., Michael, A. F., Fan, W. W., Zhou, B., \& Tsilibary, E. C. (2003). Glucose-induced changes in integrins and matrix-related functions in cultured human glomerular epithelial cells. American Journal of Physiology-Renal Physiology, 284(4), F671-F679. 
[40] Wu, K., Setty, S., Mauer, S. M., Killen, P., Nagase, H., Michael, A. F., \& Tsilibary, E. C. (1997). Altered kidney matrix gene expression in early stages of experimental diabetes. Cells Tissues Organs, 158(3), 155-165.

[41] Cai, W., Gao, Q. D., Zhu, L., Peppa, M., He, C., \& Vlassara, H. (2002). Oxidative stress-inducing carbonyl compounds from common foods: novel mediators of cellular dysfunction. Molecular Medicine, 8(7), 337-346

[42] Trachtman, H., Futterweit, S., Pine, E., Mann, J., \& Valderrama, E. (2002). Chronic diabetic nephropathy: role of inducible nitric oxide synthase. Pediatric Nephrology, 17(1), 20-29.

[43] Spirin, K. S., Saghizadeh, M., Lewin, S. L., Zardi, L., Kenney, M. C., \& Ljubimov, A. V. (1999). Basement membrane and growth factor gene expression in normal and diabetic human retinas. Current eye research, 18(6), 490-499.

[44] Evans, T., Deng, D. X., Chen, S., \& Chakrabarti, S. (2000). Endothelin receptor blockade prevents augmented extracellular matrix component mRNA expression and capillary basement membrane thickening in the retina of diabetic and galactose-fed rats. Diabetes, 49(4), 662-666.

[45] Nishikawa, T., Giardino, I., Edelstein, D., \& Brownlee, M. (2000). Changes in diabetic retinal matrix protein mRNA levels in a common transgenic mouse strain. Current eye research, 21(1), 581-587.

[46] Sorensen, V. B., Rossing, P., Tarnow, L., Parving, H. H., Noergaard, T., \& Kastrup, J. (1998). Effects of nisoldipine and lisinopril on microvascular dysfunction in hypertensive Type I diabetes patients with nephropathy. Clinical Science, 95, 709-717. 